\title{
Carla Taban, Modalités po(i)étiques de configuration textuelle: le cas de "Molloy" de Samuel Beckett
}

\section{Gianmaria Finardi}

\section{(2) OpenEdition}

1 Journals

\section{Edizione digitale}

URL: http://journals.openedition.org/studifrancesi/6435

DOI: $10.4000 /$ studifrancesi.6435

ISSN: 2421-5856

\section{Editore}

Rosenberg \& Sellier

\section{Edizione cartacea}

Data di pubblicazione: 1 novembre 2010

Paginazione: 590-591

ISSN: 0039-2944

\section{Notizia bibliografica digitale}

Gianmaria Finardi, «Carla Taban, Modalités po(i)étiques de configuration textuelle: le cas de "Molloy" de

Samuel Beckett», Studi Francesi [Online], 162 (LIV | III) | 2010, online dal 30 novembre 2015, consultato il 09 janvier 2021. URL: http://journals.openedition.org/studifrancesi/6435 ; DOI: https://doi.org/

10.4000/studifrancesi.6435

Questo documento è stato generato automaticamente il 9 janvier 2021.

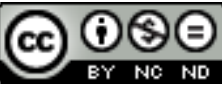

Studi Francesi è distribuita con Licenza Creative Commons Attribuzione - Non commerciale - Non opere derivate 4.0 Internazionale. 


\title{
Carla Taban, Modalités po(i)étiques de configuration textuelle: le cas de "Molloy" de Samuel Beckett
}

\author{
Gianmaria Finardi
}

\section{NOTIZIA}

CARLA TABAN, Modalités po(i)étiques de configuration textuelle: le cas de "Molloy" de Samuel Beckett, Amsterdam-New York, Rodopi, 2009 («Faux titre», 332), p. 360.

1 Con questo volume Carla Taban non intende tanto fornire un'ennesima interpretazione del Molloy francese di Beckett, bensì tentare di spiegare le ragioni intrinseche della molteplicità delle letture cui l'opera, a partire dalla sua pubblicazione, nel 1951, si è prestata. Secondo questo studio, infatti, la pluralità di significati dell'a-roman beckettiano, concepito principalmente come artefatto di lingua, risulta condizionata dal sapiente sfruttamento del medium linguistico, ossia dalla manipolazione delle possibilità contestuali-verbali di significazione del francese. In quest'ottica, il lavoro poetico effettuato sul materiale linguistico di Molloy, da un lato, e il lavoro poietico di messa in atto tramite una struttura plurisignificante, dall'altro, costituiscono le due facce di un unico processo qui designato con il termine ambivalente di po(i)eticità.

2 La po(i)eticità di Molloy si può meglio cogliere, del resto, se rapportata alla nozione di opera aperta elaborata da Umberto Eco: si tratta di svelare quell'intricato sistema di relazioni, instaurate a livello squisitamente linguistico, capace di generare una molteplicità di significati. Per circoscrivere tale sistema verbale in Molloy, vengono presi in esame i procedimenti linguistici a funzione umoristica, ironica o straniante, considerati dalla critica come il marchio di fabbrica dell'autore irlandese: sino ad ora analizzati da un punto di vista poetico come dispositivi fondamentalmente ludici, i famosi giochi di parole beckettiani vengono qui indagati per la loro portata poietica, testualmente strutturante. 
3 Dopo aver discusso i tre principali contributi critici riguardanti i giochi di parole messi in campo da Beckett, l'A. provvede a stilarne un inventario esaustivo suddiviso per tipologie; tale classificazione mostra la varietà di procedimenti utilizzati e, di contro, i limiti dell'approccio tassonomico e decontestualizzante della maggior parte degli studi sin qui svolti. Si procede quindi all'applicazione di un metodo contestualizzante che, nell'accezione strutturalista dell'epiteto, prevede l'individuazione delle peculiarità verbali del testo, evidenziate in rapporto a contesti linguistici sempre più estesi. Seguendo questa scrupolosa metodologia, si dimostra il valore po(i)etico, al tempo stesso stilistico e configurante del testo, delle espressioni idiomatiche e della metafora in generale che sono recuperate dal francese comune e risem(ant)izzate. Il medesimo valore po(i)etico si riscontra nello studio comparativo-contrastivo del Molloy francese accostato ora all'autotraduzione in inglese ora a traduzioni italiane e tedesche. L'individuazione, nella sezione conclusiva, di una rete di relazioni fondata sulla ripresa degli stessi giocosi costrutti linguistici non solo contribuisce a ribadire il fenomeno di configurazione testuale osservato, ma finisce anche per porre Molloy in rapporto diretto con altri testi non necessariamente beckettiani. 